\title{
Versatile counterstrategies shift the balance of intersexual conflict from males to females
}

\author{
Zuofu Xiang ${ }^{1}$, Yang YU ${ }^{1}$, Hui Yao ${ }^{1}$, Qinglang $\mathrm{Hu}^{2}$, and Ming $\mathrm{Li}^{3}$ \\ ${ }^{1}$ Central South University of Forestry \& Technology \\ ${ }^{2}$ Shennongjia National Park, Hubei Province \\ ${ }^{3}$ Institute of Zoology, Chinese Academy of Sciences
}

August 4, 2020

\begin{abstract}
Although primate females are predicted to develop counterstrategies against male infanticide, it has remained unclear whether counterstrategies are effective. Detailed long-term observations in Rhinopithecus roxellana reveal that females are able to mount versatile counterstrategies, including extra-unit mating, female joint defense and transfer with the infants, which lead to only 4 of 31 infants succumbing to infanticide and no difference in mortality of infants present during male replacements compare to other periods. Female joint defenses crucially delay infanticide and provide an opportunity for mothers' transfer to another social unit. In response to mothers' transfer, some males cease attacking infants, which is likely beneficial to males as during longer tenure they can sire future offspring of the mothers who remain. Our findings show how dynamic interactions between males and females can be and how the conditions in multilevel societies can shift the balance of intersexual conflict from males to females.
\end{abstract}

\section{Versatile counterstrategies shift the balance of intersexual conflict from males to females}

\section{Short title: Lowered infanticide in snub-nosed monkeys}

Zuofu Xiang ${ }^{1, *}$, Yang $\mathrm{Yu}^{1}$, Hui $\mathrm{Yao}^{2}$, Qinglang $\mathrm{Hu}^{1}$, Ming $\mathrm{Li}^{3,4}{ }^{*}$

Author affiliation:

${ }^{1}$ College of Life Science and Technology, Central South University of Forestry \& Technology, Changsha, Hunan, 410004, China

${ }^{2}$ Shennongjia National Park, Hubei Province, Shennongjia Forest District, Hubei 442411, China

${ }^{3}$ Key Lab of Animal Ecology and Conservation Biology, Institute of Zoology, Chinese Academy of Sciences, Beijing, 100101, China

${ }^{4}$ Center for Excellence in Animal Evolution and Genetics, Chinese Academy of Sciences, Kunming, 650223, China

*Correspondence author:

Zuofu Xiang

498 Shaoshan Nanlu, Changsha, Hunan, 410018, P.R. China

Institute of Evolutionary Ecology and Conservation Biology,

Central South University of Forestry \& Technology, 
Tel: 86-731-5623392; Fax: 86-731-5623498

Z Xiang (xiangzf@csuft.edu.cn)

M Li (lim@ioz.ac.cn)

Authorship: ZX and ML conceived the research design, analyzed the data, and wrote the paper jointly. ZX, HY, YY, ZZ and QH performed the field observation, and ZX and ML conducted the paternity analysis.

Keywords : Sexual conflict, male infanticide, female counterstrategies, Rhinopithecus roxellana, multi-level society, polymorphic personality

\begin{abstract}
Although primate females are predicted to develop counterstrategies against male infanticide, it has remained unclear whether counterstrategies are effective. Detailed long-term observations in Rhinopithecus roxellana reveal that females are able to mount versatile counterstrategies, including extra-unit mating, female joint defense and transfer with the infants, which lead to only 4 of 31 infants succumbing to infanticide and no difference in mortality of infants present during male replacements compare to other periods. Female joint defenses crucially delay infanticide and provide an opportunity for mothers' transfer to another social unit. In response to mothers' transfer, some males cease attacking infants, which is likely beneficial to males as during longer tenure they can sire future offspring of the mothers who remain. Our findings show how dynamic interactions between males and females can be and how the conditions in multilevel societies can shift the balance of intersexual conflict from males to females.

In sexually reproducing species, the reproductive strategies of females and males often diverge, creating conflict between the sexes (Parker, 2006). Such conflict can result in antagonistic coevolution (Chapman \& Partridge 1996; Rice 1996), where individuals of each sex are selected to maximize their fitness at the expense of their mates (Trivers 1972). Intersexual conflict has frequently been studied from the perspective of males to unravel the conditions under which strategies involving harm to females are adaptive (Chapman et al. 2003) or in some case from the perspective of females to determine the conditions leading to either successful resistance or to females gaining at the expense of males (Ebensperger 1998; Palombit 2015). However, most studies have focused on a single sex, limiting our understanding of interactions and potential dynamic switch in the use of strategies and counter-strategies.
\end{abstract}

There are many forms of sexual conflict in non-human primates, with infanticide of unweaned young by adult males the most ubiquitous example (Hrdy 1997; Palombit 2012; Yao et al. 2016): killing unrelated infants shortens the inter-birth interval of the mother, permitting the killer to increase his reproductive success by siring her next infant sooner. In some species with frequent rank or resident status change among the males up to $50 \%$ of unweaned infants might be killed by males (Yin et al. 2013; Ma et al. 2019), generating considerable costs to females. In turn, female primates exhibit a range of behavior that have been shown or suggested to be counterstrategies to avoid infant loss from male infanticide. Females might reduce the overall risk of infanticide by mating with many males, such that future dominants cannot be certain that a given offspring is not their own (Wolff \& MacDonald, 2004; Lukas \& Huchard 2014). If females are pregnant during situations when infanticide is likely to occur, such as take-overs or male rank changes, they have been found to terminate their pregnancies (Roberts et al. 2012). If females have dependent offspring during these situations, they sometimes defend their offspring by separating it spatially from the infanticidal male, by counter-attacking infanticidal males on their own or in coalition with other females or a protector male, or by leaving the social unit and the infanticidal male, transferring with the ousted male, or joining a new social unit (Palombit 2012; Roberts et al. 2012).

Even though female counterstrategies have been reported in various species, few studies investigate their effectiveness and if and how males might react. This balance in the sexual conflict likely depends on the social and mating system. For example, in gregarious species, females might benefit from having improved detection and coalitionary defense against males that might be infanticidal (Nunn \& van Schaik 2000; Zipple et al. 2017), but in itself, female coalitionary defense had been considered as ineffective because it can only 
delay infanticide (Palombit 2012). More effective strategies might be female promiscuity, for which females would need to have access to alternative mating partners in order to confuse paternity; protection from other males, which might be also limited in uni-male societies; or be tolerated by other females in order to transfer into a new group/unit, which might limited in territorial species. Female counterstrategies might be expected to be more effective if, in turn, males might gain benefits from stopping to attack infants. For example, if females would not leave a male who stopped attacking infants, this male might gain future reproductive opportunities if he can retain his tenure and mating control during future reproductions of these females. Accordingly, we would expect sexual conflict between males and females to be particular dynamic in flexible societies, where some individuals might have the opportunities to change different aspects of their behavior depending on the behavior of the individuals of the opposite sex.

Golden snub-nosed monkeys (Rhinopithecus roxellana) are Asian colobines endemic to China living in a multi-level society, in which females reside in core social units consisting primarily of one male unit (OMU) with multiple females, and multiple such OMUs associate and forage together as one larger social band (Xiang et al. 2014; Grueter et al. 2020). Infanticide has been observed when males take over the dominant position in OMUs (Yao et al. 2016), but most unweaned infants present during the times of male replacement survive without being attacked by the new male. Recently, female promiscuity has been proposed as one counterstrategy against male infanticide in golden snub-nosed monkeys (Qi et al. 2020), but detailed interactions and potential dynamic switch between males and females, i.e. female joint defense, mothers transfer with their infants and males' response to these counterstrategies, remain unclear. The nestedness of social contacts in golden snub-nosed monkeys means that females have the chance to obscure paternity as they have the opportunity for regular contact with males outside their own OMU, and extra-unit paternities have been confirmed in this species (Guo et al. 2010; Yao et al. 2016). In addition, the spatial association among and distribution of kin across units also means that females potentially have low costs of dispersal facilitating transfer between units (Qi et al. 2009; Zhang et al. 2019), but remain among close social partners which might provide support against attacks. Therefore, the social flexibility in snub-nosed monkeys might offer an opportunity to investigate the role of social interactions on the expression of male infanticide and female counterstrategies.

In this study, we firstly investigate the support for the effectiveness of female counterstrategies to male infanticide based on a 15-year, detailed observations of female and male behavior during take-overs and of the fate of fetus or infants in a free-ranging population of $R$. roxellana . We hypothesize that females' counter-strategies would be versatile and effective and mortality of unweaned infants would show no difference between infants present during times of male replacement and those present during other times. In particular, we expect that (i) pregnant females would terminate their pregnancies following male replacement with or without male aggression; (ii) females copulate with males other than the leader of their OMU, including with potential killers (unassociated adult males in the all-male unit, who would take over their OMU) or with potential protectors (resident males of other OMUs, who could offer protection for the mother and unweaned infant if they immigrate into these OMUs); (iii) if the unweaned infant suffer an unsuccessful attack from new male, females would leave their original OMUs to seek male protection, either with the ousted male or immigrating into a new OMU where they have previously mated with the male leader or where they have female relatives; (iv) females are able defend offspring through joint defense, we predict that larger size of OMU (members except the infant) would lead less risk of being killed or attacked because female joint defense might be stronger and/or early detection and vigilance might also be more efficient in larger OMU.

We next investigate strategies of males in response to the females' counterstrategies and their benefits. Considered males have two different behaviors, tolerance or aggression, towards the unweaned infant, we predict that older males are more likely to be aggressive because they need immediate reproductive success and cannot wait for future reproductive success. Furthermore, as resident males are more likely to sire the next offspring of females in their OMUs, we predict that males would benefit from tolerating or refraining to attack after lactating female transferring with unweaned infant if (i) male tenures are longer than females' birth intervals, and/or (ii) male who tolerate with unweaned infant without attack might have longer tenures than males who consistently attack unweaned infants. 


\section{MATERIALS AND METHODS}

Study sites and subjects We conducted the study at Dalongtan $\left(31^{\circ} 29^{\prime} \mathrm{N}, 110^{\circ} 18^{\prime} \mathrm{E}\right.$; elevation: $\left.2200 \mathrm{~m}\right)$ in Shennongjia National Park, central China. The study population is free-ranging, but partly provisioned. Reserve staff began provisioning the monkeys in 2006 for the purpose of ecotourism (Yu et al. 2013; Zhang et al. 2019). Food was provided two to three times per day and included lichen, pine seeds, apples, carrots, oranges, and peaches; the monkeys were provisioned at different feeding sites/platforms. When not provisioned, the monkeys ranged across an area with a radius of $1 \mathrm{~km}$. Provisioning likely affected the monkeys' social behavior and daily activities, especially early in the process. However, they soon became habituated to humans, and instead of fleeing, the monkeys started to ignore the presence of researchers and reserve staff most of the time. Therefore, observations were made on a daily basis at distances between 5 and 50 m (Xiang et al. 2019). All adult members were individually identifiable based on a unique set of physical features, such as body size, pelage color, evidence of injuries or scars, the shape and size of a female's nipples, and the shapes of granulomatous flanges which are present of fleshy nodules on both sides of the upper lip in adult males (Yu et al. 2013). The size of the focal band has increased from $\sim 30$ individuals comprising three OMUs and an associated AMU (five adult individuals) in January 2006 to $>90$ individuals in five OMUs and one AMU (> seven adult males) in December 2019.

Data collectionWe collected data on a daily basis at distances 5 - $50 \mathrm{~m}$ from 0800 to 1800 in winter-spring or 0700-1900 in summer-autumn between January 2006 and March 2020. All instances of births, abortions, copulations and male takeovers were observed using ad libitum sampling. However, once a male takeover event had taken place, male attacks on pregnant females, infants and mothers with infants, as well as any abortions, infanticide events were observed in a focal animal sampling. This focal animal sampling extended to the end of the mating (potential conception) season (late November) of the year of the takeover (Yao et al. 2016). All events were recorded via continuous all-occurrence sampling once an event began (Xiang et al. 2019). The final replacement of the previous male was decided to have occurred when the previous male left the original unit and joined the AMU or when copulations between the new male and the females were observed without disturbance by the previous male. A successful infanticide attempt was defined to have occurred when the infant was killed by the male during the first set of attack, otherwise aggressive bouts towards the infant were scored as unsuccessful infanticide attempts.

For the paternity analysis, hairs with intact roots were collected from all infants born during the study period, their mothers, and from all reproductively mature adult males in the focal band (Guo et al. 2015). Hair samples of adult individuals were obtained by plucking them directly by hand (with gloves) while samples of young infants - who were difficult to approach - were obtained by using a pole with glue on its end which was brought in contact with the back of the target individuals (Guo et al. 2015). Paternity exclusion was established using sixteen different microsatellite loci (D13S321, D10S1432, D1S533, D14S306, D6S493, D7S817, D10S676, D1S1656, D1S1665, D7S1826, D7S2204, D6S1056, D2S442, D5S1457, D6S474, D10S611) [for details on the DNA protocols see Xiang et al. (2014) and Yang et al. (2014)]. The reliability of our genotyping results from hair samples was confirmed by comparing allele patterns with matched blood samples of five captive individuals: the agreement was $99.1 \%$. Paternity for the 99 offspring born into the band from 2007 to 2018 was determined with the software CERVUS3.0 (Kalinowsk et al. 2007) with confidence levels of $>80 \%$ (relaxed confidence) and $>95 \%$ (strict confidence). These inferences matched exclusion standards, and in all cases in which paternity was assigned to an extra-unit male the male residing within the social unit during the conception was excluded at two or more loci. The relatedness of female dyads in study population was divided into three categories: all "mother-daughter" dyads and six full "sister-sister" dyads were determined by observing infants born into OMUs since 2006, and five half "sister-sister" dyads were identified based on the genetic data.

Data analysis Extra-unit copulations and extra-unit paternity were judged to have occurred if the mate or the farther resided in a different social unit. A chi-square test was used to identify whether (i) any extra-unit copulations primarily occurred between females and established males residing in one-male units or males residing in the all-male unit, and (ii) frequencies of extra-unit copulation of those female involved in male 
replacement are higher than those not involved. A Z score test was used to identify (i) if fetal or infant death rates following male replacement are higher than other circumstances, (ii) if pregnant females tend to terminate their pregnancies following male replacement. A two-sample independent $t$-test was used to establish whether there were significant differences (i) size of OMU (members except the infant) between OMUs in which infants were attacked or killed by males versus those in which infants did not suffer attacks, if (ii) between females' birth interval and males' tenure, and (iii) in the tenures between the tolerance male and aggressive male.

\section{RESULTS}

\section{Male replacement, pregnant females, unweaned infants and their fortunes}

We observed 18 male replacement events involving 16 OMUs and 15 adult males between January 2006 and March 2020 (Case \#1 - \#18,Table S1 ). Two events occurred when no females were pregnant or caring for unweaned offspring (\#7 and \#18). Eight pregnant females were present during male replacement events, four of which were attacked by new males, which subsequently resulted in two abortions (\#1 and \#3), one gave birth but the newborn was killed by the new male (\#11), and one returned to stay with ousted male and gave birth and weaned her offspring (\#17). All other pregnant females were not attacked by the new males and gave birth to their offspring.

Thirty-one unweaned infants were present following male replacements. Two of them and their mothers left the unit with the ousted male and survived. Although half of all remaining unweaned infants $(15 / 29)$ were attacked by new males, only three infants (\#2, \#8 and \#11) were killed by the first set of attack, and one more infant was killed after repeated attacks (\#8). Therefore, except of four infanticides, most unweaned infants $(27 / 31)$ present during male replacements are known to have survived throughout the mating season either because their mothers stayed with the ousted male, transferred to another social unit after an unsuccessful attack, or were tolerated by the new male (Fig 1 and Table S1) .

The difference in mortality of fetus and infants during male replacement compared to stable periods

Two abortions were observed during a total of 117 pregnancies outside of male takeover events. Therefore, there is a significant increase of abortions following male replacement $(\mathbf{F i g} \mathbf{2}, 2 / 8$ vs 2/117, Z-Score $=3.6212$, $p<0.001$ ). Twenty-three unweaned infant deaths were observed during a total of 121 births, with two infants who were present during times of male replacement dying for unknown reasons in winter (potentially because of low temperature and disease). We compared the difference of death rates between the infants and the fetus + infants present during times of male replacement and those present at other times. There is no significant difference of death rates between the infants present during times of male replacement and those present stable periods $(6 / 31$ vs $17 / 90$, Z-Score $=0.3408, p>0.05)$, and between the fetus + infants present during times of male replacement and those present stable periods $(8 / 33$ vs $19 / 92$, Z-Score $=0.7102, p>$ $0.05)$.

\section{Female counterstrategies against male infanticide}

Extra-unit mating In total, 4,697 copulation events were observed. Only 317 of these copulation events were extra-unit matings (6.7\%), and these involved almost exclusively males in other one-male units (309 events) rather than males in the all-male unit ( 8 events) $\left(\chi^{2}=182.00, p<0.001\right)$. Seven cases of extra-unit paternity, which involved resident males in other OMUs, were identified in 99 births (6.25\%). All new males who took over OMUs came from AMUs, and accordingly were not the fathers of any offspring in the OMUs they entered.

Of the nine mothers with unweaned infants who left their original OMU and transferred to another OMU following male replacement, two mothers ( $\mathrm{Ln}$ in Case \#6, Yb in case \#16) had previously been observed mating with the resident male of the destination OMU into which they transferred, but no extra-unit paternities were identified for these two cases. 
Joint defense with other femalesIn cases of male attacks on unweaned infants, most females of the same OMU $(83.1 \%, 59 / 71)$ were observed to joint with other females against the new male (Table S1 ). The lactating female whose infant was under attack was the initiator of $66.7 \%(10 / 15)$ of coalitions as she, after firstly seizing her infant, started shouting at the perpetrator. Then, other females of the same unit (and sometimes also juveniles) ran to the mother and subsequently started vocalizing loudly at the male. In the remaining instances the mother was still seizing her infant while other female already started shouting at the male before being joined by others (including the mother). In a few cases, non-lactating female collectively chased the perpetrator and keep it away. In response to females' facing and shouting at the male, he usually retreated quickly and stayed away from the females for some time.

The number of adult females involved in collective defense was $3.38 \pm 0.89$ (mean $\pm \mathrm{SD}$ ). The defensive behavior of females resulted in $85.0 \%(17 / 20)$ of the initial male attacks being unsuccessful, and since in the majority of cases mothers whose infants were attacked subsequently transferred to another OMU, most of these infants $(16 / 17,94.1 \%)$ survived to weaning. The effectiveness of female joint defense appears to depend on the size of OMU (the number of individuals except infants), as OMU size was smaller in OMUs in which attacks had been observed than in OMUs in which no attacks were observed (Fig 3a $, t=4.57, d f=26$, $p<0.001$ ), and the size of OMUs in which infants died from infanticide was also significantly smaller than those in which males never killed infants (Fig $\mathbf{3 b}, t=2.53, d f=29, p<0.05)$.

F emales' transfer with infants Nearly a third of mothers (11/31) transferred to other OMUs or returned to stay with the old (ousted) male in response to the unsuccessful attacks on their offspring or another offspring in the OMU (Fig 1 ). The contexts of the nine mothers with dependent offspring who successfully immigrated into an already existing OMU included (i) five females who had at least one close female relative (mother-daughter, sister-sister) in the new unit they joined, (ii) two females who had been observed mating with the resident male of destination unit, and (iii) two sets of two females who collectively joined another $\mathrm{OMU}$ as a clique.

\section{Males' strategies towards unweaned infant and its benefit}

After male replacement, new males showed two strategies, either aggression or tolerance, which would bring different reproductive success. When males adopted a strategy of aggression, they obtained increased reproductive success from killing infants because they sired the next offspring of victims' mother sooner (Case \#2, \#8 and \#11). However, they completely lost benefits as lactating females emigrated if the first set of attack did not kill the offspring (\#4, \#6 and \#17). Although infanticide might be expected to be favoured by older males, who might rely more on immediate fitness benefits, aggressive males are not older than tolerant males $(t=1.93, d f=14, p=0.0738)$. On the other side, when males adopted a strategy of tolerance, which means they tolerated unweaned infant without any attack (\#10, \#12 and \#13) or when lactating female transferred the males refrain from further attacks (\#5, \#9 and \# 14-16), they needed to wait longer until siring the next offspring of these females, but are likely to gain increased future reproductive benefits with those females who did not emigrate: (i) male tenures after take-over lasted on average 39.4 months, longer than the (on average) 23.5 months it takes a female to successfully wean her current offspring $(t=3.55, d f$ $=62, p<0.001$, Fig $4 \mathbf{a}$ ), and, (ii) tenures of tolerant males are significant longer than those of aggressive males $(t=2.31, d f=9, p<0.05$, Fig $4 \mathbf{b})$.

\section{DISCUSSION}

The detailed observations of female responses during male-takeovers across fifteen years suggest that living in a multi-level society means that females can mate with extra-unit males, form joint defense with other females in the same OMU against male attacks, and are able to successfully transfer to other OMUs to remove their unweaned offspring from infanticidal males. These counter-strategies appear effective, as in response most males would tolerate the dependent infant and/or stop attacking any further infants when some mothers left with infants, and thus only a small number of infants present during take-overs were killed by males (4/31). Males in turn gain from changing their own strategy, as tolerating unweaned infants means 
their mothers stay with him, and tolerant males would have a more chance of siring offspring with them in the future with longer tenure. These findings suggest that social constraints might be important in shifting the balance of sexual conflict either towards females or towards males.

In response to the threat of infanticide by males, females have been argued to exhibit the Bruce effect, where they terminate pregnancies after exposure to unfamiliar males as an adaptive strategy as it is highly likely that a female's offspring will be killed after its birth (Roberts et al. 2012). This counter-strategy has been confirmed in other wild mammals including a wild primate, the gelada (Theropithecus gelada) with $80 \%$ of pregnancies terminated in the weeks after a dominant male is replaced. In other studies of Bruce effect (Berger 1983; Hackländer \& Arnold 1999), more than $90 \%$ pregnant females induce abortions following male takeover. However, the rate of fetal death $(25 \%)$ is much lower than fetal death rates reported in those studies, suggesting that fetal loss in study population is not an obligate response to male takeovers. Therefore, abortions in golden snub-nosed monkey might be the result of sexually selected feticide rather than the Bruce effect in instances where the new males are aggressive towards females and their offspring, as has been shown in savannah baboon (Zipple et al. 2017).

In golden snub-nosed monkeys, the Bruce effect has likely failed to evolve due to several reasons. First, there is no additional benefit for females to terminate pregnancy before August. The females could not be conceptive shortly after abortions because golden snub-nosed monkeys are strictly seasonal breeders with most birth occurring from March to May and most conceptions occurring between late August and early November (Xiang et al. 2017). Second, the risk of male infanticide is relatively low because females have more versatile and effective counter-strategies. Therefore, the best evolutionary strategy is not to terminate gestation because the pregnant females have many other counter-strategies to reduce the risk of male infanticide.

Female primates appear best able to defend their offspring against male infanticide if they have the flexibility to interact with a range of social partners and rely on multiple counterstrategies. Multi-male mating by females in mammals has been indicated to be one of the most effective strategies to prevent infanticide, as it obscures paternity such that males refrain from killing offspring if they might be their father (Wolff \& MacDonald, 2004; Lukas \& Huchard 2014). In snub-nosed monkeys, extra-OMU mating and extra-OMU paternities occur between females and males residing in other OMU (Li \& Zhao 2007; Guo et al. 2010; Yang et al. 2014). Furthermore, we observed two cases where females were accepted into a unit with a resident male with whom she had previously been observed mating although there no paternities were identified; another one case was also reported in a recent study (Qi et al. 2020). In contrast, females very rarely mated with males residing in the all-male unit, even though all males taking over OMUs came from this unit because resident males from multiple OMUs collectively show intense aggression towards these outside males in golden snub-nosed monkeys (Xiang et al. 2014), presumably limiting opportunities for females to mate with them and to reduce the risk of infanticide potentially even further as it does in some polygynandrous societies.

As there is frequently pronounced dimorphism between females and males in primates, it has been suggested that mothers would be unable to prevent infanticide physically (Ebensperger 1998; Palombit 2012). In this study, we find that, while females might be unable to resist a male attack on their own, in nearly $85 \%$ of attacks females formed joint defenses that were effective in preventing males from killing their offspring in that instance. Therefore, although female joint defenses against male attacks has sometimes been considered ineffective because females might be unable to completely prevent males from killing offspring (de Waal 1997), our findings suggest that in snub-nosed monkeys it might create the crucial delay for females to exhibit other counterstrategies, in particular keeping their offspring alive until they might have the opportunities to transfer from their unit. This effect was more pronounced in larger units, potentially because more adult females in an $\mathrm{OMU}$ can lead to stronger female-female coalitions, vigilance and risk detection might also be more efficient (Palombit 2012).

By transferring from their social unit mothers can remove their unweaned offspring from a potential killer male (Hrdy 1997). Establishing a new unit with the ousted male, as was observed in four cases here, is 
presumably more likely to occur in species in which the habitat is not saturated with groups that defend territories. Alternatively, females can attempt to transfer into an already established social unit where a male would be unlikely to kill the offspring (Steenbeek et al. 1997). In multi-level societies such as in golden snub-nosed monkeys, the potential costs of transferring into a new unit are likely to be reduced compared to other societies. Some females already disperse as sub-adults, and both natal and secondary dispersal of females mostly occurs into other social units within their natal band (Guo et al. 2015). This means that females frequently have female relatives residing in neighbor OMUs, which might help them to join and be tolerated in these units. Some females have sexual experienced with other resident male int the band, this might also offer her and her unweaned infant a refuge when there is high risk of infanticide. In addition, food competition among the band member in this species is low because ubiquitous lichens and leaves form a major dietary constituent throughout the year ( $\mathrm{Li} 2006$ ), facilitating units to be in close proximity to potentially reduce predatory attacks through travelling, feeding, and resting within the same areas (Grueter et al. 2020).

The relative low costs associated with female transfers appear to influence male behavior, too. From the perspective of males taking over a social unit, emigration of adult females means that they would lose reproductive opportunities completely. While infanticide might increase current reproductive success if its mother remains in the OMU, it appears that on average males are likely to gain higher fitness if they refrain from attacking the unweaned infant. It is in the case in the study band when they do not kill their current offspring because male tenures are significant longer than the female reproductive cycle, tolerant males even have longer tenure than those aggressive males, and males are likely to sire future offspring of females as the low probability of extra-unit paternity. Similarly, a resident male of an OMU might not only accept an immigrating lactating female because he previously mated with her, but also because he can increase his future reproductive success if he remains dominant for at least another reproductive cycle of that female.

Additionally, we confirm that females snub-nosed monkeys are able to mount versatile and effective counterstrategies against male infanticide including extra-unit mating, joint defense and transfer, leading to no significant difference in the mortality of infant present during male replacement to the base-line mortality of infants in stable groups (Fig 2). Furthermore, mortality from infanticide for those infants involved male replacement in the study population was also much lower than the mortality observed in some Asian colobines in which females live in single-male or multi-male societies. For example, in an uni-male society of white-headed langurs (Trachypithecus leucocephalus ), all eleven infants younger than 6 months died of possible infanticide after male takeovers (Yin et al. 2013). In uni-male and multi-male society of hanuman langurs (Winkler et al. 1984; Sommer 1987; Borries 1997), death rates of infant following male replacement were $37.9 \%(11 / 29)$, and $21.6 \%$ (8/37) which also higher than golden snub-nosed monkeys $(12.9 \%, 4 / 31)$. These data might implicate that social organization or mating system would have an influence on the efficacy of female counterstrategies against infanticide.

Finally, we want provide a tentative explanation for why older males are not likely to kill the dependent infant although it would obviously increase their fitness, they might have not enough life span to wait another reproductive cycle of lactating female as those younger males. We argued that it might be explained by the male's personality which often termed as consistent individual differences in behavior across time and contexts. Based on the insight that the trade-off between current and future reproduction, animal personalities could be given an adaptive explain in polymorphic population (Wolf et al. 2007). In snub-nosed golden monkeys of a multilevel society, where multiple OMUs coordinate and forage together as one larger social band (Grueter et al. 2020), social complexity would allow polymorphic personality of resident male in the band. In this study, we have observed two kinds of male personalities towards unweaned infants following male replacement. An aggressive male would obviously obtain immediate reproductive success by infanticide, and this personality may not only benefit to the females in his OMU when competed with patch resources (i.e., one tree with more fruit or better for sleeping) but also benefit to the population when confronted a predator because more aggression to conspecific also mean bolder in interspecific fight to a predator in many species (Sih et al. 2004). A tolerant male would obviously obtain future reproductive success through longer tenures (Fig $\mathbf{4 b}$ ) and monopolize paternity by attracting females' residency. Therefore, aggressive males are 
favored by natural selection, and tolerance males might favor by sexual selection in this multilevel society. In fact, we have observed an extreme example which show the male's personality might be stable. For instance, in case \#8 and \#17, the male (NN) continue attacking the dependent infant which result two infants were killed (Case \#8) or all two lactating females emigration (Case \#17). In the first case, the male obtained current and future reproductive success, however, he lost all lactating females in later case.

Ethics

All research methods adhered to the Chinese legal requirements and were evaluated and approved by Central South University of Forestry \& Technology's Institutional Animal Care and Use Committee (IACUC) (protocol \#2012-018).

\section{Acknowledgments}

We thank Penglai Fan, Haochun Chen, Hanlong Chen, Ruoshuang Liu, Bo Zhang, Li Wei, Lang Lu and help collect data in the field. We thank Professor Tim Clutton-Brock and Dr Dieter Lukas for his constructive comments on this paper. This study was supported by Supported by the Strategic Priority Research Program of Chinese Academy of Sciences (XDB31000000), the National Natural Science Foundation of China (31870509, 31670397, 31821001),National Key R\&D Program of China (2016YFC0503200) and the State Forestry Administration of China.

\section{Competing interests}

The authors declare that they have no competing interests.

\section{Data and materials availability}

The datasets supporting this article have been uploaded as part of the electronic supplementary material.

\section{REFERENCES}

Berger, J. (1983). Induced abortion and social factors in wild horses.Nature 303 , 59-61.

Borries, C. (1997). Infanticide in seasonally breeding multimale groups of Hanuman langurs (Presbytis entellus) in Ramnagar (South Nepal). Behav. Ecol. Sociobio. 41, 139-150.

Chapman, T. \& Partridge L. (1996). Sexual conflict as fuel for evolution. Nature381, 189-190.

Chapman, T., Arnqvist, G., Bangham, J. \& Rowe, L. (2003). Sexual conflict. Trends Ecol. Evol. 18 , 41-47.

Ebensperger, L.A. (1998). Strategies and counterstrategies to infanticide in mammals. Biol. Rev. 73 , $321-346$.

Grueter, C.C., Qi, X., Zinner, D., Bergman, T., Li, M., Xiang, Z., et al . (2020). Multilevel organisation of animal sociality. Trends Ecol. Evol . doi:10.1016/j.tree.2020.05.003.

Guo, S., Huang, K., Ji, W. \& Li, B. (2015). The role of kinship in the formation of a primate multilevel society. Am. J. Phys. Anthropol. 156, 606-613.

Guo, S., Ji, W., Li, M., Chang, H. \& Li, B. (2010). The mating system of the Sichuan snub-nosed monkey (Rhinopithecus roxellana).Am. J. Primatol. 72, 25-32.

Hacklander, K. \& Arnold, W. (1999). Male-caused failure of female reproduction and its adaptive value in alpine marmots (Marmota marmota). Behav. Ecol. 10 , 592-597.

Hrdy, S.B. (1997). The Langurs of Abu: Female and Male Strategies of Reproduction. (Harvard University Press, Cambridge, MA).

Kalinowski, S.T. \& Taper, M.L. \& Marshall, T.C. (2007). Revising how the computer program CERVUS accommodates genotyping error increases success in paternity assignment. Mol. Ecol. 16, 1099-1106. 
Li, B. \& Zhao, D. (2007). Copulation behavior within one-male groups of wild Rhinopithecus roxellana in Qinling Mountains of China.Primates , 48 , 190-196.

Li, Y.M. (2006). Seasonal variation of diet and food availability in a group of Sichuan snub-nosed monkeys in Shennongjia Nature Reserve, China. Am. J. Primatol. 68, 217-233.

Lukas, D. \& Huchard, E. (2014). The evolution of infanticide by male in mammalian societies. Science 346 , 841-844.

Ma, C.Y., Brockelman, W.Y., Light, L.E. O., Bartlett, T.Q. \& Fan, P.F. (2019). Infant loss during and after male replacement in gibbons.Am. J. Primatol. 81, e 23036.

Nunn, C.L. \& van Schaik, C.P. (2000). Social evolution in primates: The relative roles of ecology and intersexual conflict. In:Infanticide by Males and Its Implications (eds by van Schaik, C.P. \& Janson, C.H.) pp. 388-420.

Palombit, R.A. (2012) Infanticide: Male strategies and female counterstrategies. In: Evolution of Primate Societies (eds Mitani, J.C., Call, J.P., Kappeler, P.M., Palombit, R.A. \& Silk, J.B.), pp. 432-468.

Palombit, R.A. (2015). Infanticide as Sexual Conflict: Coevolution of Male Strategies and Female Counterstrategies. Cold Spring Harb Perspect Biol 7 , a017640.

Parker, G. A. (2006). Sexual conflict over mating and fertilization: an overview. Philos. Trans. R. Soc. B. 361 , 235-259.

Qi, X., Li, B., Garber, P.A., Ji, W. \& Watanabe, K. (2009). Social dynamics of the golden snub-nosed monkey (Rhinopithecus roxellana ): female transfer and one-male unit succession. Am. J. Primatol. $\mathbf{7 1}$, 670-679.

Qi, X.G., Grueter, C.C., Fang, G., Huang, P., Zhang, J., Duan, Y.,et al. (2020). Multilevel societies facilitate infanticide avoidance through increased extrapair matings, Animal Behav, https://doi.org/10.1016/j.anbehav.2019.12.014.

Rice, W.R. (1996). Sexually antagonistic male adaptation triggered by experimental arrest of female evolution. Nature $\mathbf{3 8 1}, 232-243$.

Roberts, E.K., Lu, A., Bergman, T.J. \& Beehner, J.C. (2012). A Bruce effect in wild geladas. Science 335, 1222-1225. Sih, A., Bell, A. \& Johnson, J.C. (2004). Behavioral syndromes: an ecological and evolutionary overview. Trends Ecol. Evol.19, 372-378. Sommer, V. (1987). Infanticide among free-ranging langurs (Presbytis entellus) at Jodhpur (Rajasthan/ India): Recent observations and a reconsideration of hypotheses. Primates 28, 163-197.

Steenbeek, R., Piek, R.C., van Buul, M. \& vanHoff, J.A.R.A.M. (1999). Vigilance in wild Thomas's langurs (Presbytis thomasi ): the importance of infanticide risk. Behav. Ecol. Sociobiol.45, 137-150.

Trivers, R. (1972). Parental investment and sexual selection. In: Sexual Selection and the Descent of Man ( eds Campbell, B.) pp. 136-179.

de Waal, F.B.M. (1997). Bonobo: The Forgotten Ape. (University of California Press, Berkeley). Winkler, P. Loon, H. \& Vogel, C. (1984). Life history of Hanuman langurs (Presbytis entellus). Reproductive parameters, infant mortality, and troop development. Folia Primatol. 43, 1-23. Wolf, M., van Doorn, G.S., Leimar, O. \& Weissing, F.J. (2007). Life-history trade-offs favour the evolution of animal personalities.Nature 447, 581-584.

Wolff, J.O. \& MacDonald, D.W. (2004). Promiscuous females protect their offspring. Trends Ecol. Evol. $19,127-134$.

Xiang, Z.F., Fan, P., Chen. H., Liu, R., Zhang, B., Yang, W., et al. (2019). Routine allomaternal nursing in a free-ranging Old World monkey. Sci. Adv. 5, eaav0499. 
Xiang, Z.F., Yang, B., Yu, Y., Yao, H., Grueter, C.C., Garber, P.A.et al . (2014). Males collectively defend their one-male units against bachelor males in a multi-level primate society. Am. J. Primatol. 76, 609-617.

Xiang, Z.F., Yang, W., Qi, X., Yao, H., Grueter, C.C., Garber, P. A.et al . (2017). An examination of factors potentially influencing birth distributions in golden snub-nosed monkeys (Rhinopithecus roxellana). PeerJ 5, e2892.

Yang, B. Ren, B., Xiang, Z., Yang, J., Yao, H., Garber, P.A., et al. (2014) Major histocompatibility complex and mate choice in the polygynous primate: the Sichuan snub-nosed monkey (Rhinopithecus roxellana). Integrat. Zool. 9 , 598-612.

Yao, H., Yu, H., Yang, B., Yang, W., Xu H., Grueter, C.C., Li, M. \& Xiang Z. (2016). Male infanticide in the golden snub-nosed monkey (Rhinopithecus roxellana), a seasonally breeding primate.Int. J. Primatol. 37, 175-184. Yin, L., Jin, T., Watanabe, K., Qing, D., Wang, D. \& Pan, W. (2013). Male attacks on infants and infant death during male takeovers in wild white-headed langurs (Trachypithecus leucocephalus).Integrat. Zool. 8, 365-377.

Yu, Y., Xiang, Z., Yao, H., Grueter, C.C. \& M. Li. (2013) Female snub-nosed monkeys exchange grooming for sex and infant handling.PloS one $\mathbf{8}$, e74822.

Zhang, Y.J., Chen. Y., Chen, H., Yao, H., Yang, W., Ruan, X., et al. (2019). Social functions of relaxed mouth-opening display in golden snub-nosed monkeys (Rhinopithecus roxellana). Zool. Res.40, 89-96.

Zipple, M.N., Grady, J.H., Gordon, J.B., Chow, L.D., Archie, E.A., Altmann, J. \& Alberts, S.C. (2017). Conditional fetal and infant killing by male baboons.Proc. R. Soc. B 284, 20162561.

\section{SUPPLEMENTARY MATERIALS}

Supplementary material for this article is available at http://

Table S1 showing male attacking and fortune of unweaned infant, outcome of adult female with unweaned infant after male takeover.

\section{Figure captions}

Fig 1 The fortune of unweaned infants (UIs) ofRhinopithecus roxellana following male takeovers: of 31 unweaned infants that were present during male replacement, 27 infants survived and 4 infants died of male infanticide during the study period 2006 and 2020. (Number of females/infants observed showing a particular behavior are shown in circles).

Fig 2 Variations in fetal and infant death rates during the male replacement. Statistically significant increases were seen fetal death rate following male replacement. No parallel increase was seen in death of infant and fetal +infant, indicating an evidence for hypothesis that a multilevel society of golden snub-nosed monkeys facilitate female counterstrategies against male infanticide.

Fig 3 Differences in size of social unit (one male unit, OMU) with and without recorded of male attacks on infants and infanticide indicating an evidence for hypothesis that larger OMU size can lead to less risk of being killed or attacked because stronger female-female joint defense and vigilance of risk detection might also be more efficient.

Fig 4 After male replacement, new males showed two strategies, either aggression or tolerance, which would bring different reproductive success. When males adopted a strategy of aggression, they obtained increased reproductive success from killing infants because they sired the next offspring of victims' mother sooner. However, they completely lost benefits as lactating females emigrated if the first set of attack did not kill the offspring. Therefore, male would benefit from their strategies because (a) male tenures are longer than inter-birth intervals of female if keeping mothers and their unweaned infant in their social unit, (b) tenures of tolerant male are longer tenures than those of aggressive male if the male tolerate the unweaned infant without attack. 


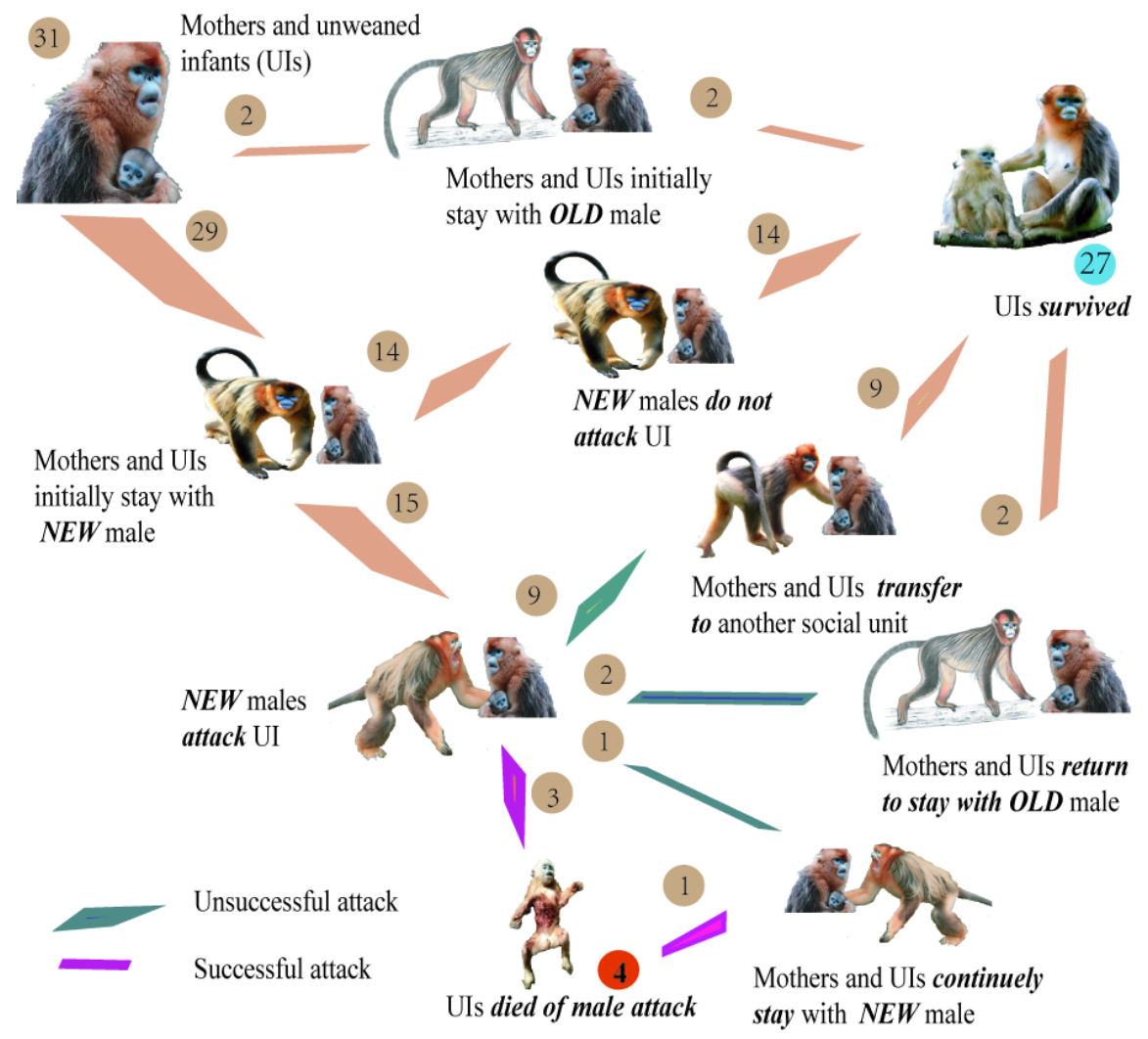

Fig 1 The fortune of unweaned infants (UIs) of Rhinopithecus roxellana following male takeovers: of 31 unweaned infants that were present during male replacement, 27 infants survived and 4 infants died of male infanticide during the study period 2006 and 2020. (Number of females/infants observed showing a particular behavior are shown in circles). 


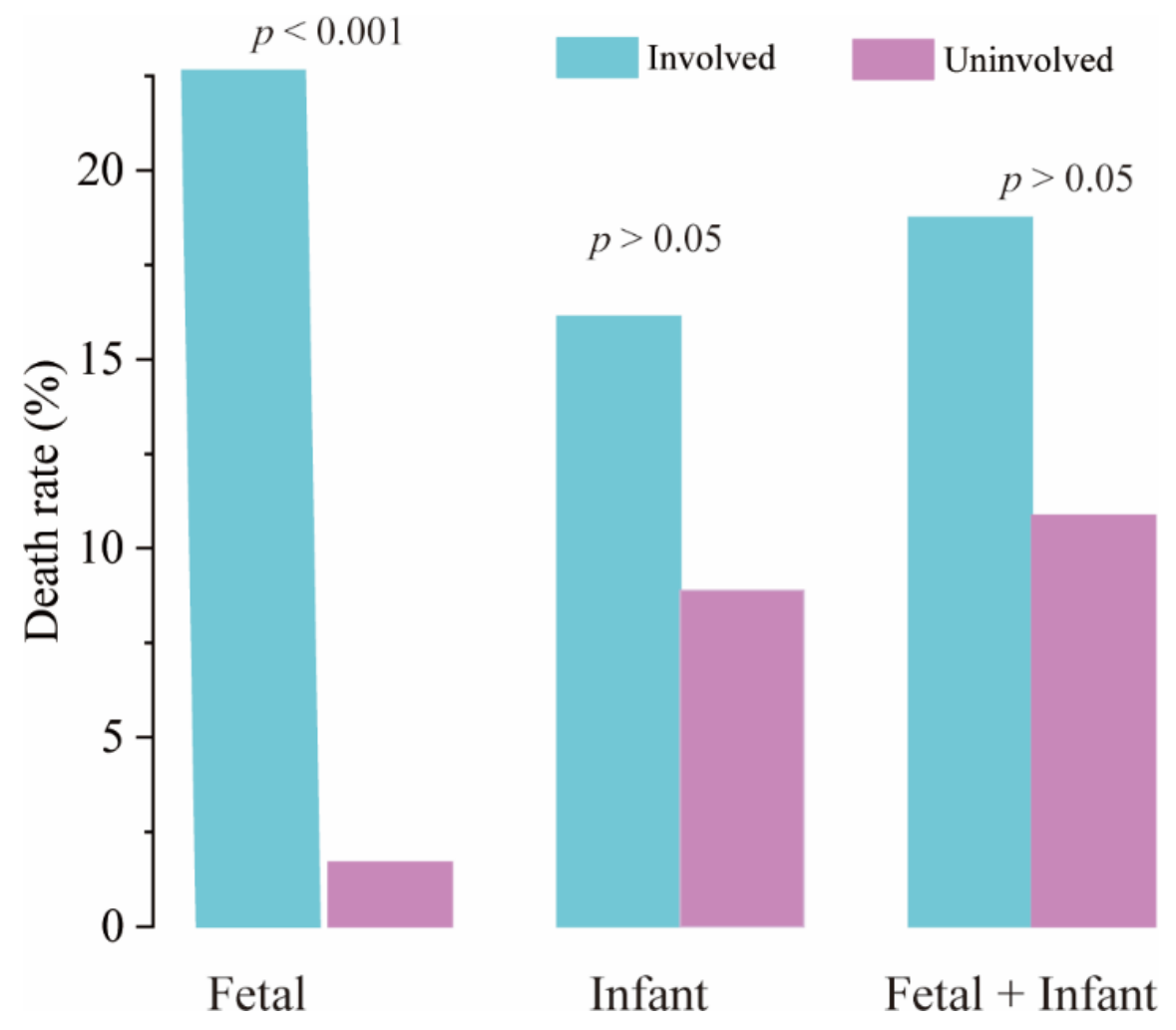

Fig 2 Variations in fetal and infant death rates during the male replacement. Statistically significant increases were seen fetal death rate following male replacement. No parallel increase was seen in death of infant and fetal +infant, indicating an evidence for hypothesis that a multilevel society of golden snub-nosed monkeys facilitate female counterstrategies against male infanticide. 


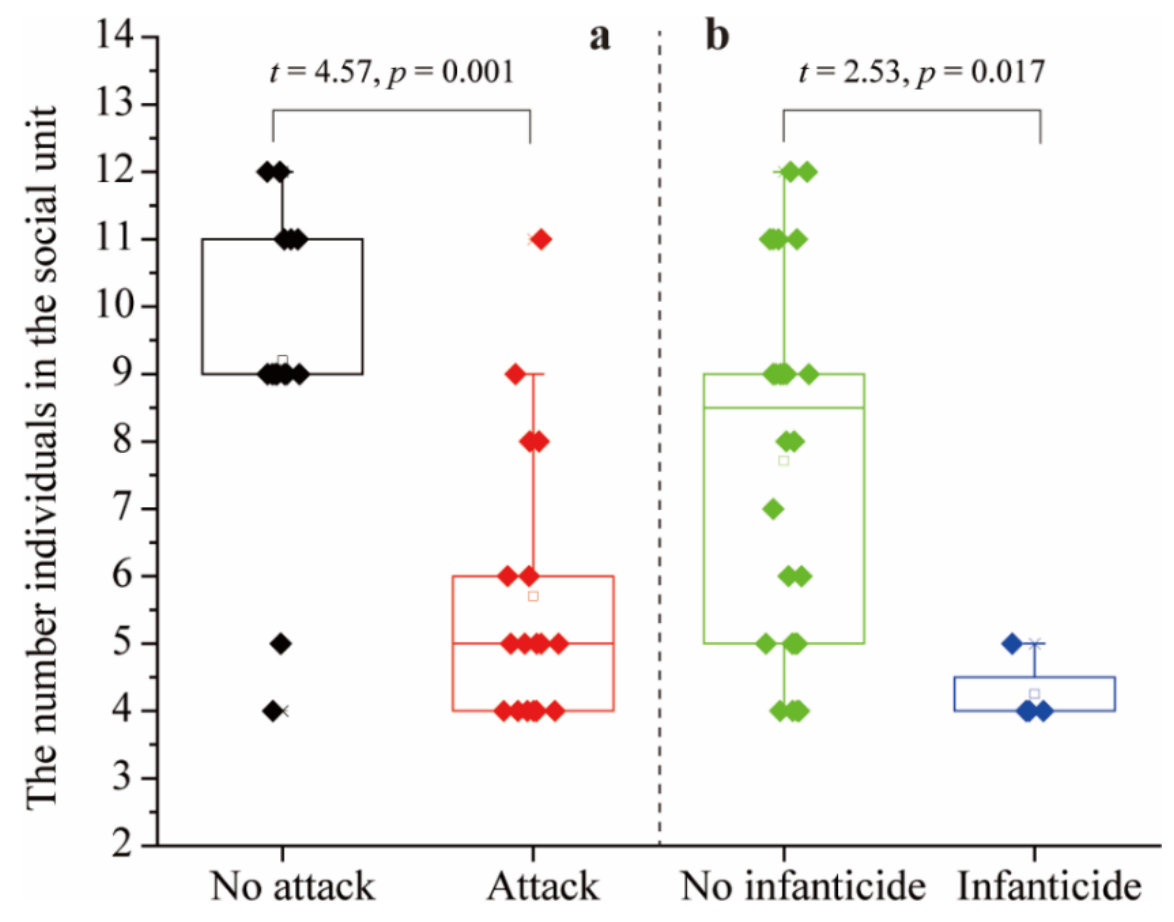

Fig 3 Differences in size of social unit (one male unit, OMU) with and without recorded of male attacks on infants and infanticide indicating an evidence for hypothesis that larger OMU size can lead to less risk of being killed or attacked because stronger female-female joint defense and vigilance of risk detection might also be more efficient.

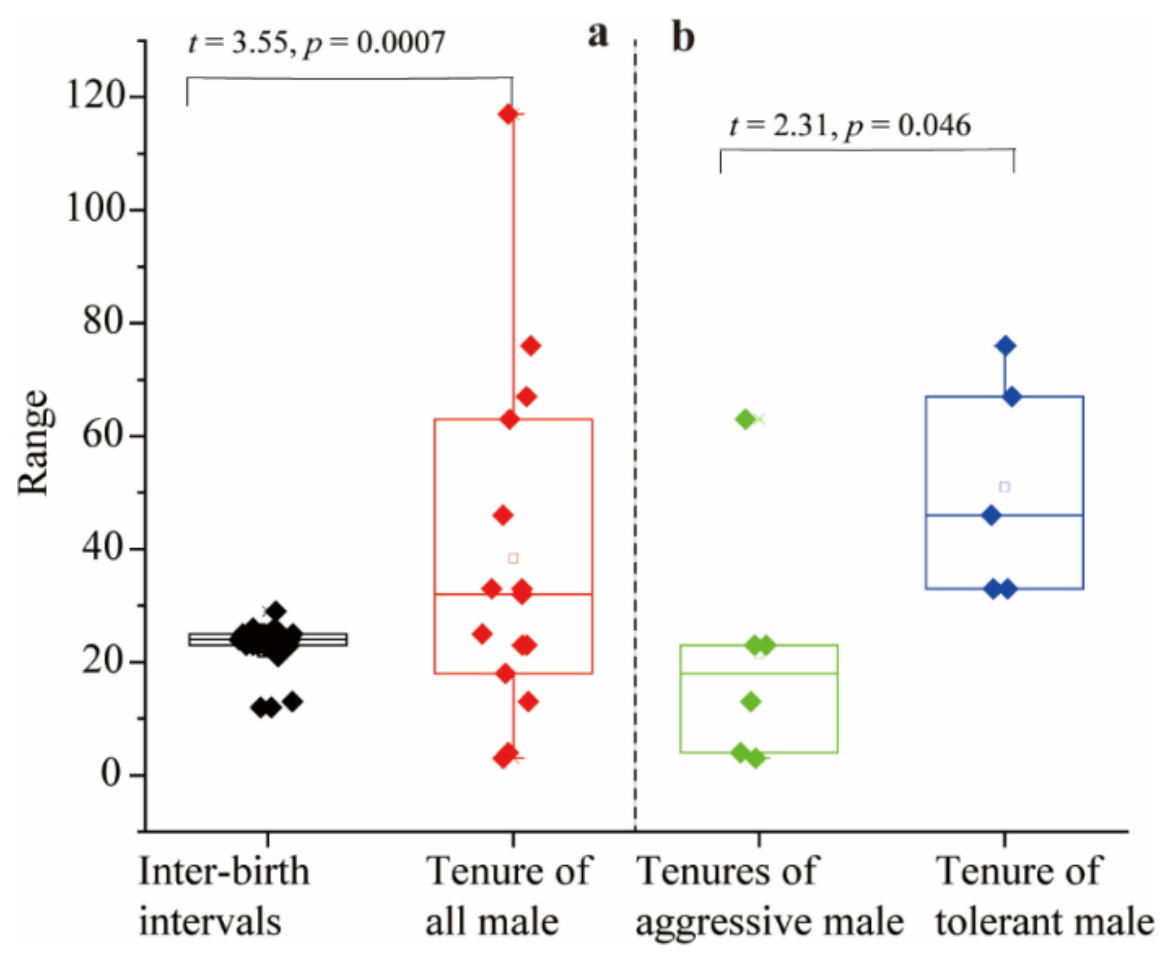


Fig 4. After male replacement, new males showed two strategies, either aggression or tolerance, which would bring different reproductive success. When males adopted a strategy of aggression, they obtained increased reproductive success from killing infants because they sired the next offspring of victims' mother sooner. However, they completely lost benefits as lactating females emigrated if the first set of attack did not kill the offspring. Therefore, male would benefit from their strategies because (a) male tenures are longer than inter-birth intervals of female if keeping mothers and their unweaned infant in their social unit, (b) tenures of tolerant male are longer tenures than those of aggressive male if the male tolerate the unweaned infant without attack.

Table S1 Male attacking and fortune of pregnant female, unweaned infant, outcome of mothers, and unweaned infant follo

\section{Events}

\#1

\#2

\#3

\#4

\#5

\#6

\#7

\#8

\#9

\#10

\#11

\#12

\#13

\#14

\#15

\#16

\#17

\#18 
Table S1 Male attacking and fortune of pregnant female, unweaned infant, outcome of mothers, and unweaned infant follo

The number of adult females involved collective defense are presented in brackets which are not included the repeatedly att , "New male" means the mother and unweaned infant stay with; "Old male" means the ousted male;" Transfer" means the 1 , The infant died without male attack during the winter. 